\title{
Warm sparse-dense wave inhibits cartilage degradation in papain-induced osteoarthritis through the mitogen-activated protein kinase signaling pathway
}

\author{
MUNAN LIN ${ }^{1 *}$, YANHONG LIN ${ }^{2 *}$, XIHAI LI ${ }^{3}$, WENNA LIANG ${ }^{4}$, SHUILIANG WANG ${ }^{5}$, \\ JIANSHENG LIU ${ }^{3}$, XIANXIANG LIU ${ }^{3}$, LIDIAN CHEN ${ }^{6}$ and YIN QIN ${ }^{1}$
}

${ }^{1}$ Department of Traditional Chinese Medicine, Fuzhou General Hospital of Nanjing Military Command,

Fuzhou, Fujian 350025; ${ }^{2}$ Department of Internal Medicine, Shanghang Hospital, Shanghang, Fujian 364200;

${ }^{3}$ Institute of Bone Diseases, Academy of Integrative Medicine; ${ }^{4}$ Research Base of Traditional Chinese Medicine Syndrome,

Fujian University of Traditional Chinese Medicine, Fuzhou, Fujian 350122; ${ }^{5}$ Laboratory Department,

Fuzhou General Hospital of Nanjing Military Command, Fuzhou, Fujian 350025; ${ }^{6}$ Institute of Rehabilitation Medicine,

Fujian University of Traditional Chinese Medicine, Fuzhou, Fujian 350122, P.R. China

Received September 29, 2016; Accepted June 2, 2017

DOI: $10.3892 / \mathrm{etm} .2017 .4984$

\begin{abstract}
Cartilage degradation is an important in the pathogenesis of osteoarthritis (OA). Abnormal activation of the mitogen-activated protein kinase (MAPK) signaling pathway in chondrocytes promotes an inflammatory response, resulting in the release of chondral matrix-degrading enzymes that accelerate the degradation of cartilage. As a non-pharmaceutical and non-invasive physical therapy regimen, warm sparse-dense wave (WSDW) has been successfully used for the treatment of OA. However, it remains unclear whether WSDW inhibits cartilage degradation in OA through the MAPK signaling pathway. The present study investigated the effects of WSDW on papain-induced OA in rat knee joints. Papain-induced OA was established in rats, which were subsequently divided into a model group and three experimental groups that received a WSDW with the following ratios: WSDW=1:1, WSDW=1:2 and WSDW=2:1. After 12 weeks of treatment, cartilage degradation was evaluated by Mankin scoring of paraffin-embedded sections stained with hematoxylin and eosin. The changes in cartilage structure were observed by transmission electron microscopy, and the expressions of RAS, extracellular signal-regulated kinase (ERK), p38 and p53 were measured by reverse transcription-quantitative
\end{abstract}

Correspondence to: Professor Yin Qin, Department of Traditional Chinese Medicine, Fuzhou General Hospital of Nanjing Military Command, Outpatient Building, 156 Xierhuanbei Road, Fuzhou, Fujian 350025, P.R. China

E-mail:1mnan_fzzyy@126.com

*Contributed equally

Key words: osteoarthrosis, signaling pathway, cartilage, warm sparse-dense wave chondrocyte polymerase chain reaction and western blot analysis. WSDW was demonstrated to improve the arrangement of collagen fibers, inhibit the tidemark replication and delay cartilage degradation in papain-induced OA. The expressions of RAS, ERK, p38 and p53 in the WSDW (1:2) and (2:1) groups were significantly decreased when compared with the model group $(\mathrm{P}<0.01)$. Furthermore, amongst the WSDW groups, the inhibitory effects of the WSDW (1:2) group were typically greater than those of the WSDW (1:1) and (2:1) groups. The results indicate that WSDW may inhibit cartilage degradation in papain-induced OA in rat knee joints by regulating the MAPK signaling pathway.

\section{Introduction}

Osteoarthritis (OA) is among the most prevalent chronic diseases to occur in adults, particularly in elderly patients, and is characterized by cartilage degradation (1). The most common risk factors for OA include age, previous joint injury, obesity, sex and mechanical factors such as malalignment and abnormal joint kinematics during ambulation. OA leads to the loss of tissue integrity and function (2). Therefore, the inhibition of cartilage degradation may be a potential strategy for the treatment of OA. The mitogen-activated protein kinase (MAPK) signaling pathway, composed of serine/threonine kinases, is a major pathway in the process of cartilage degradation (3). In particular, the pathway is involved in the proliferation, apoptosis and differentiation of chondrocytes, and the inflammatory response functions of a variety of physiological processes that are directly and indirectly related to inflammatory factors (4). This includes mechanical stress stimulation that occurs via intracellular signaling pathways, signal transmission to all types of transcription factors and regulation of the pathological process of cartilage degradation (5-7). Signals of the MAPK signaling pathway are transmitted from cell surface receptors to a variety of effectors, which regulate pathways governing cell differentiation, proliferation and programmed 
cell death (8). Furthermore, scaffold proteins are an increasingly recognized component of MAPK signaling and other signaling pathways (9). While these have no direct function in catalysis, they may act as platforms upon which signaling complexes assemble (10). As numerous scaffolds are spatially restricted within cells, this class of molecules may contribute to, and in some cases define, compartmentalized signaling. Furthermore, the MAPK cascade is a major intracellular signal transduction system, which includes p38, extracellular signal-regulated kinase (ERK) and multiple members of the c-Jun N-terminal kinase family (11). In addition, the MAPK cascade may be important in the onset of OA, and thus may be a useful target in the treatment of OA $(12,13)$. A previous study by our group demonstrated that warm sparse-dense wave (WSDW) upregulated the mRNA of p21 and B-cell lymphoma-2, downregulated the mRNA of p53, inhibited chondrocyte apoptosis and delayed the degradation of articular cartilage (14). However, the mechanisms underlying the effects of WSDW on the apoptosis of chondrocytes via the MAPK signaling pathway remain to be elucidated. Therefore, using a papain-induced OA in rat knee joints, the present study evaluated the effect of WSDW on cartilage degradation and the potential underlying mechanisms involving MAPK signaling.

\section{Materials and methods}

Animals. A total of 50 2-month-old Sprague-Dawley (SD) rats 260-290 g [license no. SCXK (Min) 2012-0001] that were specific-pathogen-free (SPF) and half male and half female were purchased from Fujian Medical University Laboratory Animal Co., Ltd. (Fuzhou, China). All animals were kept in a 12-h light/dark cycle, in a humidity $(56 \pm 5 \%)$ and temperature $\left(22 \pm 1^{\circ} \mathrm{C}\right)$ controlled environment and maintained on a $0.3 \%$ sodium diet. The Experimental Animal Center of Fuzhou General Hospital (Fuzhou, China) provided SPF medical laboratory animal environmental facilities. The experimental protocol was ethically approved by the Scientific Research Committee of Fuzhou General Hospital.

Experimental design. Following adaptive feeding for 1 week, all rats were randomly divided into control $(n=10$, regular feeding) and treatment $(n=40)$ groups according to a 50:50 ratio of male:female rats. The rats in the treatment group received a $12 \mu \mathrm{l}$ intra-articular injection of $1 \mathrm{U} / \mathrm{ml}$ L-cysteine-activated-papain in phosphate-buffered saline (Sigma-Aldrich; Merck KGaA, Darmstadt, Germany) in the bilateral knee joints at 1,4 and 7 days to induce OA, as previously described (15). At 2 weeks after the final injection of papain, the rats in the treatment group were randomly divided into four groups in each group $(n=10)$, namely a model group (regular feeding) and three experimental groups that received a WSDW with the following ratios: WSDW=1:1, WSDW=1:2 and WSDW=2:1. All animals of the treatment groups in the waking state were restrained on an operating platform, and the WSDW machine (XX-hx01LB; Fujian Province Xiangxing Electronic Technology Co., Ltd., Fuzhou China) covered the surface of the knee joint. The treatment groups were then treated for $30 \mathrm{~min} /$ day for 12 weeks, after which the animals were sacrificed. The changes in cartilage structure were observed by optical microscopy and transmission electron microscopy (TEM; H-7650, Hitachi, Ltd., Tokyo, Japan).

Gross morphology of the knee joints. After 12 weeks the animals were sacrificed and the tibial plateau cartilage was immediately isolated from the surface of the knee joint, then prepared with the dimensions $1 \times 1 \times 3 \mathrm{~mm}$ for TEM. The specimens were fixed with $3 \%$ glutaraldehyde and $1.5 \%$ paraformaldehyde solution $\left(\mathrm{pH} \mathrm{7.3)}\right.$ at $4^{\circ} \mathrm{C}$ for $24 \mathrm{~h}$, washed with $0.1 \mathrm{M}$ sodium cacodylate buffer and post-fixed with $1 \%$ osmic acid and $1.5 \%$ potassium hexacyanoferrate (II) solution $(\mathrm{pH} 7.3)$ at $4^{\circ} \mathrm{C}$ for $2 \mathrm{~h}$. The specimens were then dehydrated in dehydrated with graded alcohol (50, 70, 80, 90 and $100 \%$ ), and embedded in Epon-Araldite resin. All specimens were observed by TEM, as previously described (16). Briefly, ultrathin sections $(50 \mathrm{~nm})$ were cut on a Leica ultramicrotome and stained with $2 \%$ aqueous uranyl acetate, counterstained with $0.3 \%$ lead citrate at $4^{\circ} \mathrm{C}$ overnight and examined by TEM.

For optical microscopy, the isolated tibial plateau cartilage was sectioned into samples of $0.5 \times 0.5 \times 0.5 \mathrm{~cm}$ in size. The specimens were then fixed with $40 \mathrm{~g} / 1$ formalin (Sigma-Aldrich; Merck KGaA) for 3 days at room temperature, and then decalcified for 2 weeks in buffered $12.5 \%$ EDTA (Sigma-Aldrich; Merck KGaA). The samples were embedded in paraffin, sectioned at a thickness of $10 \mu \mathrm{m}$ and stained with $0.5 \%$ hematoxylin for $5 \mathrm{~min}$ and $1 \%$ eosin for $1 \mathrm{~min}$ at room temperature (17). Using ordinary optical microscopy, layers of articular cartilage and subchondral bone changes were visualized. The cartilage histological changes were evaluated according to the Mankin scoring system (18).

Western blot analysis. Total protein was extracted from the tibial plateau cartilage using RIPA buffer [50 mM Tris- $\mathrm{HCl}$ (pH 7.4), $150 \mathrm{mM} \mathrm{NaCl}, 1 \% \mathrm{NP}-40,0.5 \%$ sodium deoxycholate] with centrifugation at $20,000 \times \mathrm{g}$ at $4^{\circ} \mathrm{C}$ for $15 \mathrm{~min}$. The protein concentrations were determined by the BCA protein assay (Beyotime Institute of Biotechnology, Shanghai, China), and heated to $100^{\circ} \mathrm{C}$ for $5 \mathrm{~min}$. An equal amount of protein $(20 \mu \mathrm{g})$ per lane was separated by $12 \%$ SDS-PAGE and transferred onto PVDF membranes. The membranes were blocked for $1 \mathrm{~h}$ at room temperature with $5 \%$ non-fat milk (19), and incubated with primary antibodies against ERK (ab54230, 1:250), p38 (ab31828, 1:1,000), p53 (ab1431, 1:500), RAS (ab52939, 1:500) (all from Abcam, Cambridge, UK) and $\beta$-actin (8457, 1:1,000; Cell Signaling Technology, Inc., Beverly, MA, USA), then washed in TBST. The membranes were then incubated with a goat anti-rabbit horseradish peroxidase-conjugated secondary antibody IgG (1:10,000, ZB-2301; Zhongshan Golden Bridge Biotechnology, Co., Ltd., Beijing, China) for $2 \mathrm{~h}$ at room temperature. Immunoreactive proteins were visualized using Western Blot Chemiluminescence Luminol Reagent (Santa Cruz Biotechnology, Inc., Dallas, TX, USA), and bands were quantitated with a Bio-Rad ChemiDoc XRS+ imaging system (Bio-Rad Laboratories, Inc., Hercules, CA, USA). All data were collected from at least 3 independent experiments.

Reverse transcription-quantitative polymerase chain reaction $(R T-q P C R)$. Cartilage was obtained from the knee joints of SD 

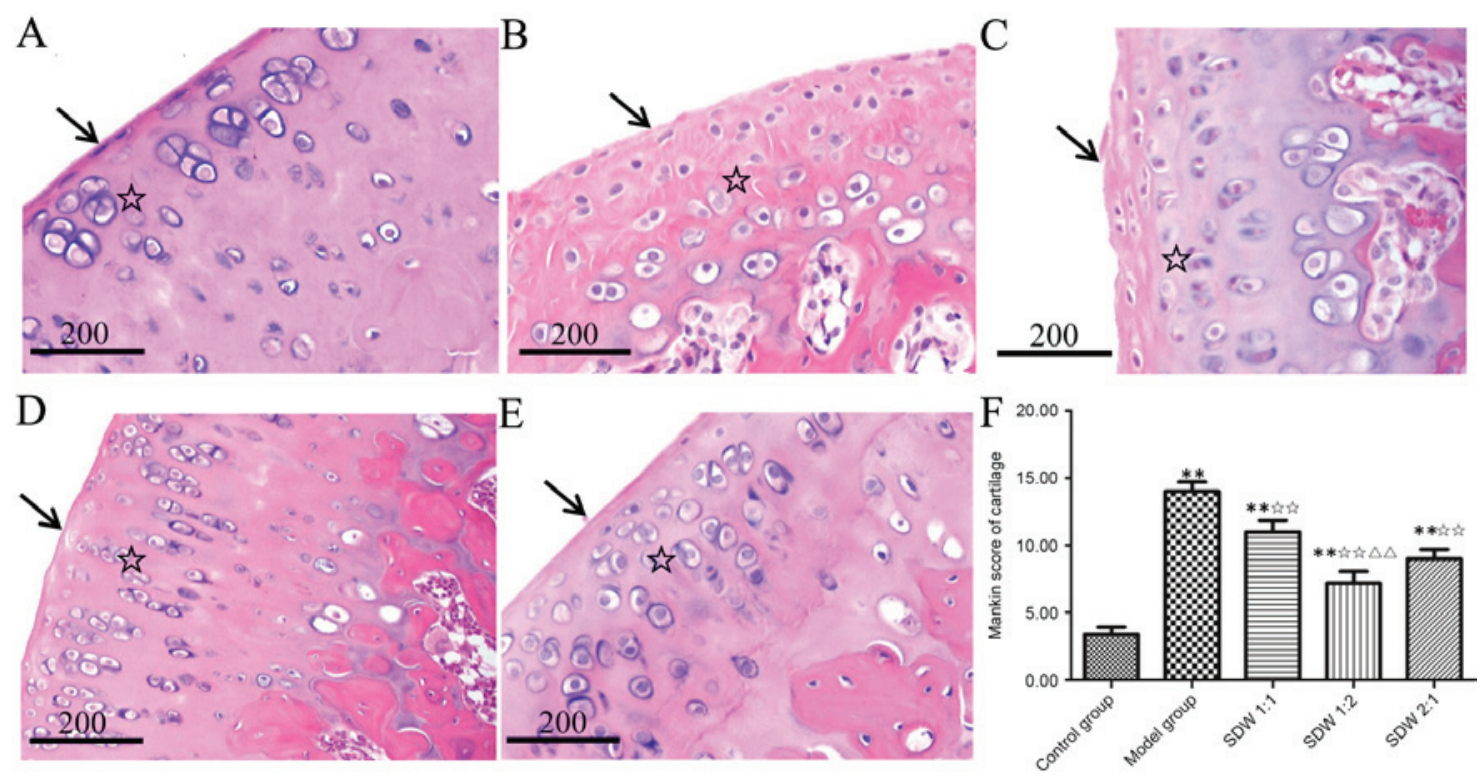

Figure 1. Histological analysis of the medial tibial plateau treated with WSDW. (A) The control group exhibited uniform surface of articular cartilage (arrow) and orderly transitional layer cells (star). (B) The model group exhibited exposed surface of articular cartilage (arrow) and disordered transitional layer cells (star). (C) The WSDW (1:1) group exhibited a loss of surface of articular cartilage (arrow) and disordered transitional layer cells (star). (D) The WSDW (1:2) group exhibited uniform surface cells (arrow) and an orderly distribution of transitional layer cells (star). (E) The WSDW (2:1) group exhibited partial repair of surface of articular cartilage (arrow) and transitional layer cells (star). Cartilage structures were detected with hematoxylin and eosin stain. Scale bar, $200 \mu \mathrm{m}$. (F) The Mankin scores of the cartilage in all groups. ${ }^{* *} \mathrm{P}<0.01$ vs. control group; ${ }^{{ }^{*} \boldsymbol{x}} \mathrm{P}<0.01$ vs. model group; ${ }^{\Delta \Lambda} \mathrm{P}<0.01$ vs. WSDW (1:1) group. WSDW, warm sparse-dense wave.

rats after treatment for 12 weeks. Total RNA was isolated using TRIzol reagent (Invitrogen; Thermo Fisher Scientific, Inc., Waltham, MA, USA) following the manufacturer's instructions. RNA $(1 \mu \mathrm{g})$ was reverse transcribed into cDNA using a reverse transcription kit (K1622; Thermo Fisher Scientific, Inc.) according to the manufacturer's instructions. qPCR was performed in a separate $20 \mu \mathrm{l}$ reaction mixture, including $1 \mu \mathrm{l}$ cDNA, $0.25 \mu 1$ Taq DNA polymerase (Invitrogen; Thermo Fisher Scientific, Inc.), $2 \mu \mathrm{l}$ primers, $2 \mu \mathrm{l}$ dNTPs, $2.5 \mu 110 \mathrm{X}$

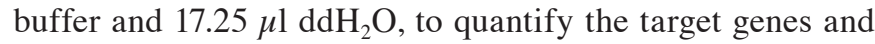
GAPDH using an ABI Prism 7500 Sequence Detection system (Applied Biosystems; Thermo Fisher Scientific, Inc.). PCR cycling conditions for each amplicon were as follows: $95^{\circ} \mathrm{C}$ for $30 \mathrm{sec}$, followed by 40 cycles of amplification consisting of denaturation at $95^{\circ} \mathrm{C}$ for $5 \mathrm{sec}$ and extension at $60^{\circ} \mathrm{C}$ for $34 \mathrm{sec}$. The PCR primers used were as follows: For ERK forward, 5'-GGC ATC CGA GAC ATC CTC AG-3' and reverse, 5'-TAT GTA CTT GAG GCC CCG GA-3'; for p38 forward, 5'-GAA TGG AAG AGC CTG ACC TAC GAT-3' and reverse, 5'-AGA GGC ACT TGA ATG GTA TTT GGA G-3'; for p53 forward, 5'-CCA TCA TCA CGC TGG AAG AC TC-3' and reverse, 5'-TGG TGG GCA GTG CTC TCT TTG-3'; for RAS forward, 5'-GGA CAA TCG CTA ACA ACC CCC T-3' and reverse, 5'-GGC ACT CTT TCC CAC GCC TCT A-3'; and for GAPDH forward, 5'-ACG GCA AGT TCA ACG GCA CAG-3' and reverse, 5'-GAA GAC GCC AGT AGA CTC CAC GAC-3'. The levels of target gene expression were normalized to that of GAPDH using the ABI Prism 7500 Sequence Detection system and the SYBR Florescence Quantization kit (Invitrogen; Thermo Fisher Scientific, Inc.). Fold-changes were calculated using the $2^{-\Delta \Delta \mathrm{Ct}}$ method (20). All data were collected from at least 3 independent experiments.
Statistical analysis. All data are presented as the mean \pm standard deviation. Data were analyzed using SPSS software version 13.0 for Windows (SPSS, Inc., Chicago, IL, USA). Statistical analysis of the data was performed by ANOVA followed by Tukey's post hoc test. $\mathrm{P}<0.05$ was considered to indicate statistically significant differences.

\section{Results}

WSDW delays cartilage degradation in papain-induced OA. In the knee joints, significant gross morphological changes were observed between the control and treatment groups (Fig. 1). The four layers of cartilage, in the control group, including the tangential zone, intermediate zone, calcified cartilage and subchondral bone, exhibited a clear articular cartilage structure and uniform cartilage matrix. Furthermore, the surface cells exhibited a fusiform and uniform distribution with neat rows of cells indicated by the cell nuclei (Fig. 1A). The cartilage in the model group exhibited disordered structure in the four layers of cartilage and damage to the articular cartilage surface. Nuclear pycnosis and necrosis was also observed in the chondrocyte area (Fig. 1B). The articular cartilage of the WSDW (1:2) group exhibited some diffuse hypercellularity and improvement in cellularity and cellular organization of cartilage compared with the model group (Fig. 1D). The pathological changes of articular cartilage in the WSDW (1:1) and (2:1) groups were improved compared with the WSDW (1:2) group but worse compared with the model group (Fig. 1C and E). The cartilage Mankin scores in the model and WSDW (1:1), (1:2) and (2:1) groups were significantly higher than those in the control group $(\mathrm{P}<0.001$, $\mathrm{P}<0.001, \mathrm{P}=0.002$ and $\mathrm{P}<0.001$, respectively), while the 
A

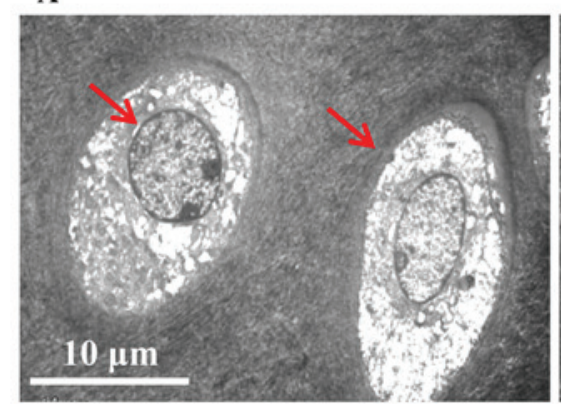

D
B

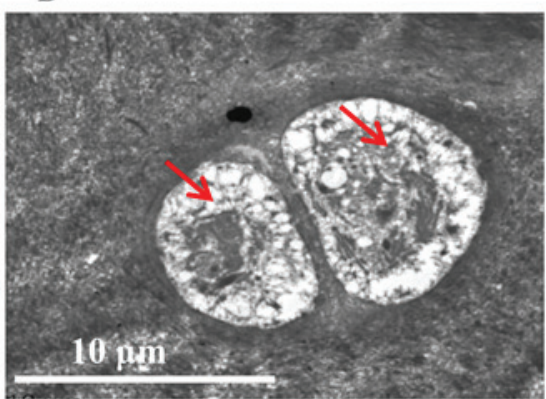

C

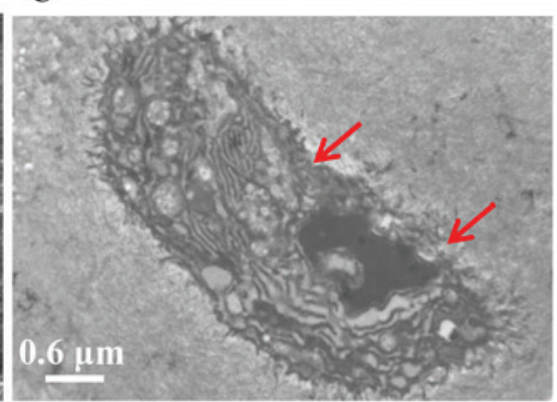

E
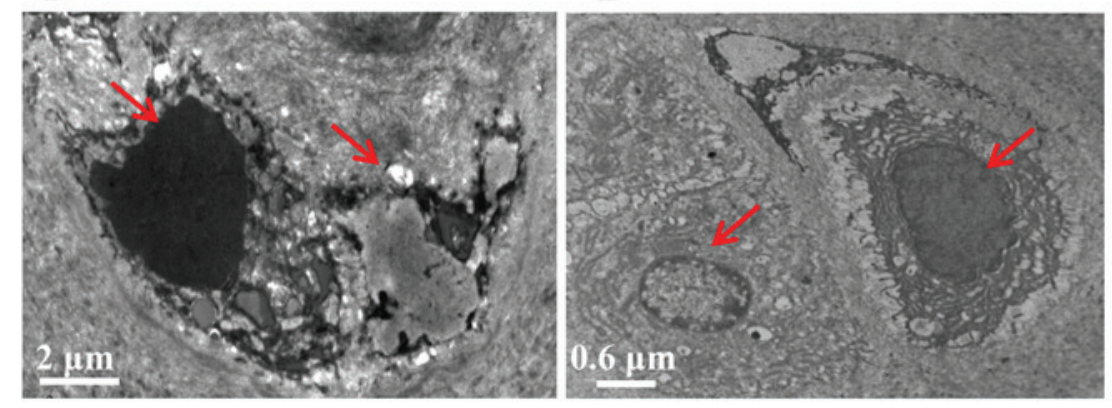

Figure 2. Analysis of the ultrastructural characteristics of the tibial plateau treated with WSDW. Cartilage ultrastructures were stained with $2 \%$ aqueous uranyl acetate, counterstained with $0.3 \%$ lead citrate. (A) Control, (B) model, (C) WSDW (1:1), (D) WSDW (1:2) and (E) WSDW (2:1) groups. Red arrows indicate chondrocytes. WSDW, warm sparse-dense wave.

Mankin scores in the WSDW (1:1), (1:2) and (2:1) groups were significantly lower than those in the model group $(\mathrm{P}=0.009$, $\mathrm{P}<0.001$ and $\mathrm{P}<0.001$, respectively). Notably, the Mankin scores in the WSDW (1:2) group were lower than those in the WSDW (1:1) group ( $\mathrm{P}=0.002$; Fig. 1F).

WSDW inhibits the degradation of ultrastructural cartilage. Ultrastructural evaluation of the tibial plateau cartilage in all groups using TEM identified differences in the treatment groups when compared with the control group (Fig. 2). In the control group, the chondrocytes were of similar size and shape with round central nuclei, uniform chromatin and visible nucleoli. In addition, the cytoplasm was rich in rough endoplasmic reticulum and the extracellular matrix collagen fibers exhibited an orderly distribution (Fig. 2A). In the model group, the tibial plateau exhibited apoptotic chondrocytes undergoing nuclear blebbing and shrinkage, along with numerous degraded collagen fibers (Fig. 2B). In comparison, cell shrinkage, endoplasmic reticulum levels, mitochondria swelling and levels of metachromatic granules in the nuclei were improved in the cartilage of the WSDW (1:2) group (Fig. 2D). Furthermore, The ultrastructural changes of articular cartilage in the WSDW (1:1) and (2:1) groups were improved compared with the WSDW (1:2) group but worse compared with the model group (Fig. 2C-E).

WSDW decreases the expressions of RAS, ERK, p38 and $p 53$. To evaluate the potential inhibitory effects of WSDW on the MAPK signaling pathway, the expressions of RAS, ERK, p38 and p53 were measured by qPCR and western blot analysis. The mRNA expression of RAS in the model and WSDW (1:1), (1:2) and (2:1) groups was significantly higher than that in the control group $(\mathrm{P}<0.001, \mathrm{P}<0.001, \mathrm{P}=0.005$ and $\mathrm{P}<0.001$, respectively). In addition, RAS mRNA expression was significantly lower in the SDW (1:1), (1:2) and (2:1) groups when compared with the model group $(\mathrm{P}=0.009$, $\mathrm{P}<0.001$ and $\mathrm{P}<0.001)$. Notably, RAS mRNA expression was significantly decreased in the WSDW (1:2) and (2:1) groups when compared with the WSDW (1:1) group ( $\mathrm{P}<0.001$ and $\mathrm{P}<0.001)$. Furthermore, the WSDW (1:2) group exhibited significantly lower expression of RAS mRNA than the WSDW (2:1) group $(\mathrm{P}=0.028)$ (Fig. 3A). The mRNA expressions of ERK, p38 and p53 in the model group and WSDW (1:1) group were significantly increased when compared with those in the control group $(\mathrm{P}<0.001, \mathrm{P}<0.001, \mathrm{P}<0.001$ and $\mathrm{P}<0.001$, $\mathrm{P}=0.002, \mathrm{P}=0.003$, respectively). In turn, all three WSDW groups exhibited significantly lower mRNA expression of p38 and p53 compared with the model group $(\mathrm{P}<0.001)$. The WSDW (1:2) and (2:1) groups also exhibited significantly lower mRNA expression of ERK mRNA compared with the model group $(\mathrm{P}<0.001)$. Furthermore, the WSDW (1:2) and (2:1) groups exhibited significantly lower expressions of ERK mRNA when compared with the WSDW $(1: 1)$ group $(\mathrm{P}<0.001$, $\mathrm{P}<0.001)$, and the WSDW (1:2) group exhibited significantly lower expression of p53 mRNA when compared with the WSDW (1:1) group ( $\mathrm{P}=0.005$; Fig. 3B-D).

As demonstrated in Fig. 4, the protein levels of RAS, ERK, p38 and p53 were similar to their respective mRNA expression levels. The protein level of RAS in the model and WSDW (1:1), (1:2) and (2:1) groups was significantly higher compared with the control group $(\mathrm{P}<0.001, \mathrm{P}<0.001, \mathrm{P}=0.014$ and $\mathrm{P}<0.001$, respectively; Fig. 4B). RAS protein level was significantly lower in the WSDW (1:2) and (2:1) groups when compared with the model group and WSDW $(1: 1)(\mathrm{P}<0.001)$. Notably, the WSDW (1:2) group exhibited significantly lower levels of RAS protein level than the WSDW (2:1) group $(\mathrm{P}=0.025)$. The protein level 

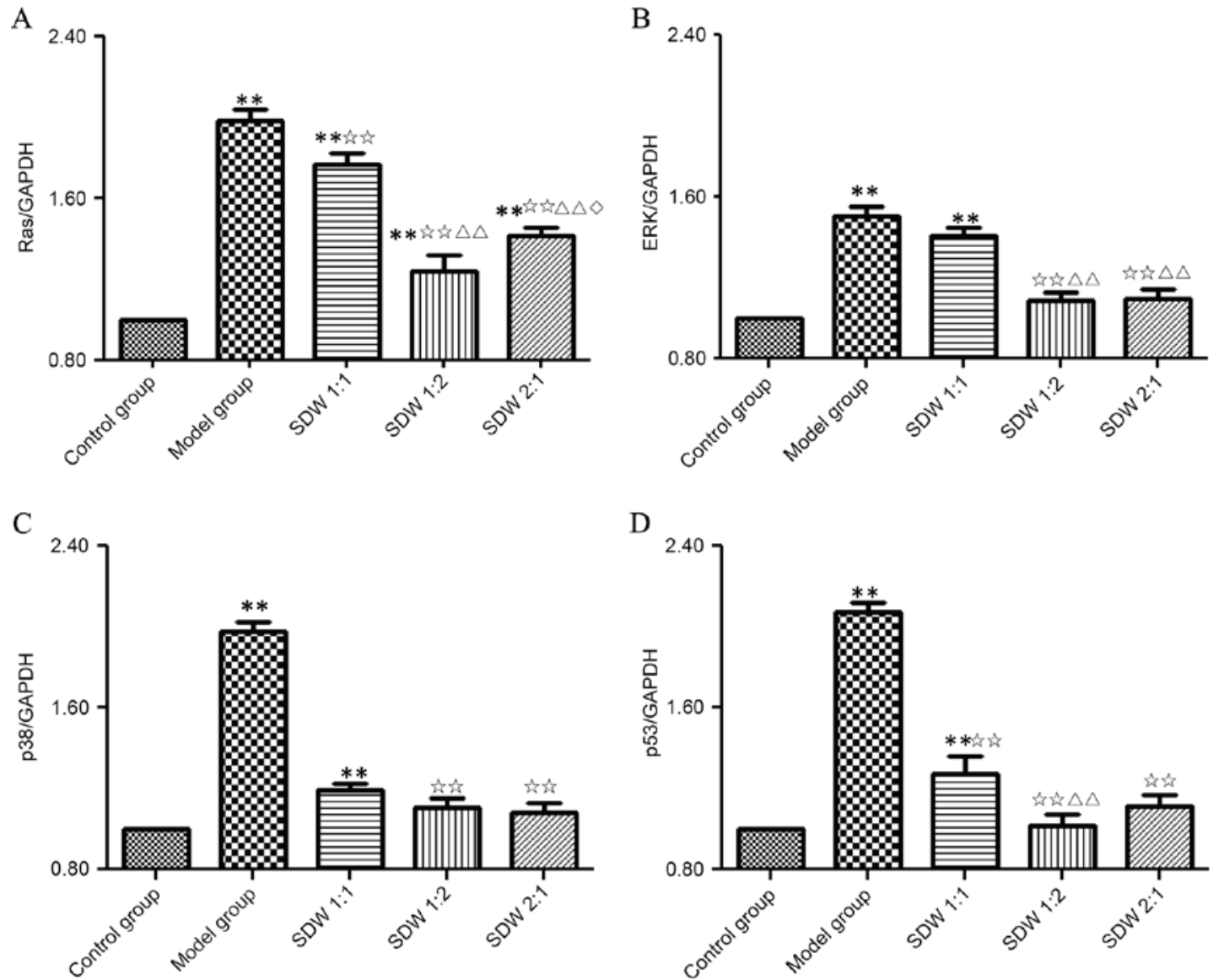

Figure 3. WSDW decreased the expression levels of RAS, ERK, p38 and p53 mRNA. The mRNA expression of (A) RAS, (B) ERK, (C) p38 and (D) p53 were measured by quantitative polymerase chain reaction analysis. GAPDH was used as an internal control for normalization. Values are presented as the mean \pm standard deviation. ${ }^{* *} \mathrm{P}<0.01$ vs. control group; ${ }^{\wedge}{ }^{\wedge} \mathrm{P}<0.01$ vs. model group; ${ }^{\Delta \Delta} \mathrm{P}<0.01$ vs. WSDW $(1: 1)$ group; ${ }^{\wedge} \mathrm{P}<0.05$ vs. WSDW (1:2) group. ERK, extracellular signal-regulated kinase; WSDW, warm sparse-dense wave.

of ERK, p38 and p53 in the model group was significantly higher than that in the control group $(\mathrm{P}=0.002, \mathrm{P}<0.001$ and $\mathrm{P}<0.001$; Fig. 4C-E). ERK protein level was significantly lower in the WSDW (1:2) and (2:1) groups when compared with the model group ( $\mathrm{P}=0.002$ and $\mathrm{P}=0.005$; Fig. $4 \mathrm{C})$. The $\mathrm{p} 38$ and p53 protein level were significantly lower in the WSDW (1:1), (1:2) and (2:1) groups when compared with the model group $(\mathrm{P}<0.001$; Fig. 4D and E). These results indicated that WSDW may delay cartilage degradation in papain-induced OA through the inhibition of RAS, ERK, p38 and p53 expressions.

\section{Discussion}

Chondrocyte apoptosis leads to cartilage degradation, and are considered to be key in the pathological development of knee OA (21). Therefore, inhibition of chondrocyte apoptosis may be effective for the treatment of OA (22-24). The results of the present study suggested that WSDW inhibited papain-induced OA by regulating the MAPK signaling pathway, as indicated by the downregulation in RAS, ERK, p38 and p53 following WSDW. This indicates that WSDW may be a potential therapeutic agent for the treatment of OA.

$\mathrm{OA}$ is a chronic illness and there is currently no radical therapy available. In addition, only a few drugs, including non-steroidal anti-inflammatory drugs and cyclooxygenase 2 inhibitors, are used to treat OA (25), and a number of adverse effects have been associated with these drugs (26-28). Traditional Chinese Medicine (TCM), including acupuncture and Chinese herbal medicine, has been demonstrated to effectively alleviate pain for thousands of years, particularly pain associated with joint diseases such as OA (29-31). An increasing number of patients are now turning to alternative and complementary medicine due to the side effects and limitations of conventional therapies. Therefore, novel therapeutic methods need to be developed to reduce the impacts of OA.

According to a TCM theory, OA is a bone obstruction disease, in which the knee joints undergo pain, stiffness and/or malfunction, consequently resulting in the syndromes of 'blood stasis' and 'cold dampness' $(32,33)$. WSDW has been widely used as an effective, safe and non-pharmaceutical therapy regimen for the management of OA, and is based on the theory of TCM acupuncture and meridian theory, suggesting that WSDW works via electrical stimulation of knee joint points (12).

The MAPK signaling pathway serves an important role in the regulation of the inflammatory response, and may be affected by a variety of inflammatory factors, such as interleukin-1 and tumor necrosis factor- $\alpha$ (34). With regard to OA, p53 inhibits DNA replication and terminates cell replication, which may lead to cell apoptosis and accelerated cartilage degradation (35). In addition, high-level expression of ERK 
A

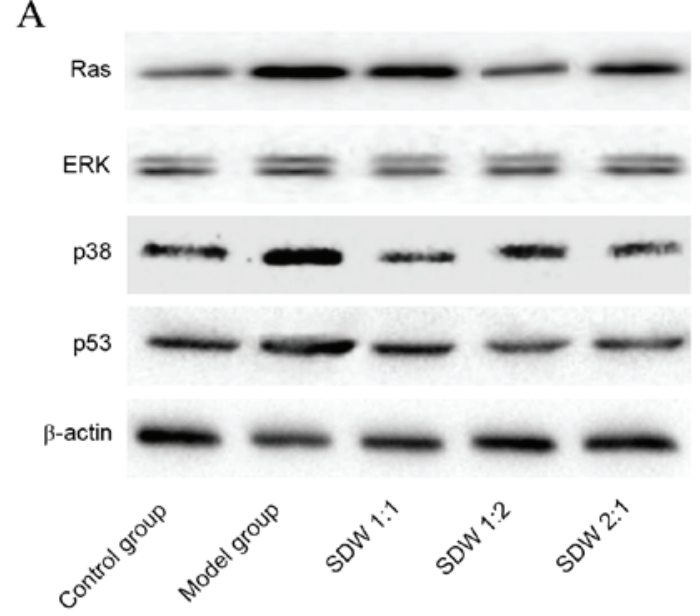

$\mathrm{C}$

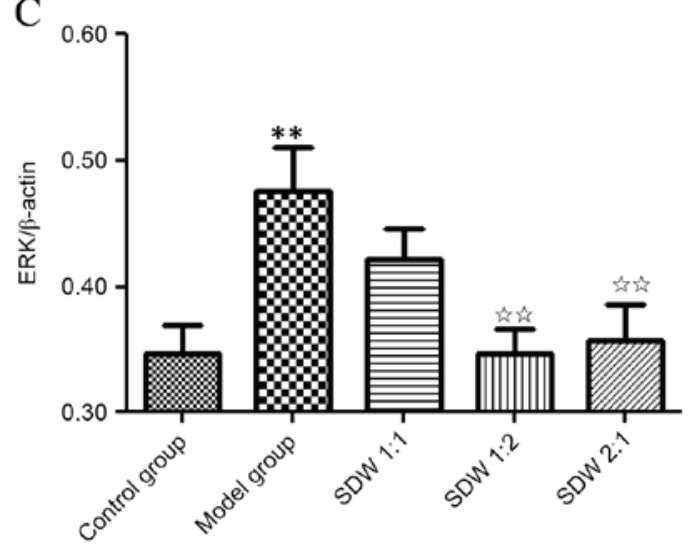

B

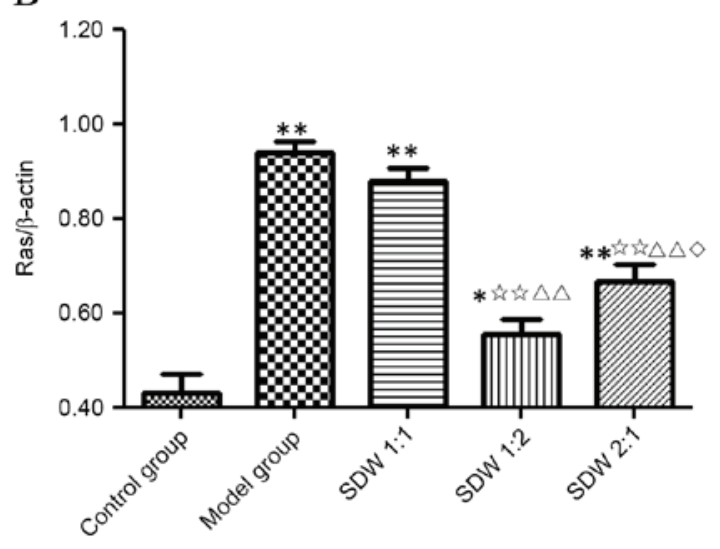

$\mathrm{D}$

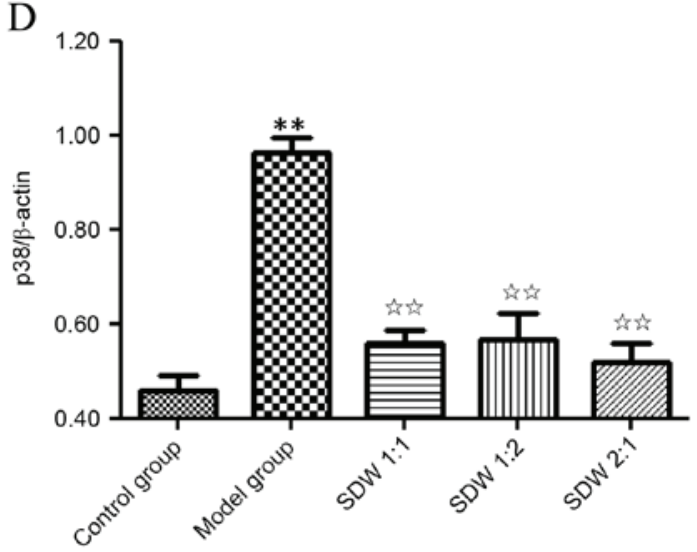

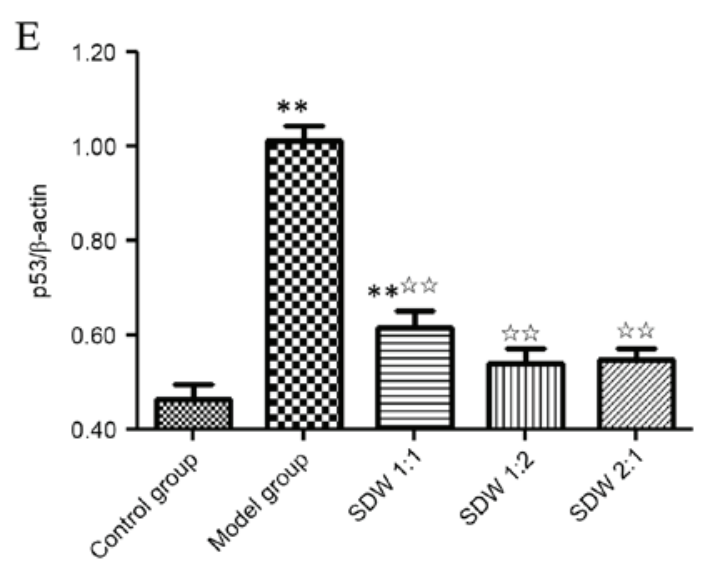

Figure 4. WSDW decreased the protein levels of RAS, ERK, p38 and p53 protein. (A) The protein levels of RAS, ERK, p38 and p53 were measured by western blot analysis. (B-E) $\beta$-actin was used as an internal control for normalization. Values are presented as the mean \pm standard deviation. ${ }^{* *} \mathrm{P}<0.01 \mathrm{vs}$. control group; ${ }^{\wedge \lambda \vec{x}} \mathrm{P}<0.01$ vs. model group; ${ }^{\Delta \Delta} \mathrm{P}<0.01$ vs. WSDW (1:1) group; ${ }^{\circ} \mathrm{P}<0.05$ vs. WSDW (1:2) group. ERK, extracellular signal-regulated kinase; WSDW, warm sparse-dense wave.

has been reported in OA (36). Furthermore, activated RAS, upon stimulation from extracellular signals, may activate ERK in the regulation of cell proliferation, differentiation, apoptosis and inflammation, and has been associated with OA cartilage degradation (37).

The results of the present study demonstrated that WSDW inhibited the expressions of RAS, ERK, p38 and p53, indicating that prompt WSDW may inhibit the MAPK signaling pathway and reduce inflammation-induced chondrocyte apoptosis. However, the present results are only applicable to the rat model of papain-induced OA, and the clinical efficacy of WSDW in humans remains to be investigated in larger samples and randomized controlled clinical trials.

\section{Acknowledgements}

The present study was supported by the Natural Science Foundation of Fujian Province (grant no. 2013J01349) and the Developmental Fund of Fuzhou General Hospital of Nanjing Military Command (grant no. 201003). 


\section{References}

1. Harrison C, Henderson J, Miller G and Britt H: The prevalence of diagnosed chronic conditions and multimorbidity in Australia: A method for estimating population prevalence from general practice patient encounter data. PLoS One 12: e0172935, 2017.

2. Hart HF, Collins NJ, Ackland DC, Cowan SM, Hunt MA and Crossley KM: Immediate effects of a brace on gait biomechanics for predominant lateral knee osteoarthritisand valgus malalignment after anterior cruciate ligament reconstruction. Am J Sports Med 44: 865-873, 2016

3. Wang Z, Guo A, Ma L, Yu H, Zhang L, Meng H, Cui Y, Yu F and Yang B: Docosahexenoic acid treatment ameliorates cartilage degeneration via a p38 MAPK-dependent mechanism. Int J Mol Med 37: 1542-1550, 2016.

4. Shi J, Zhang C, Yi Z and Lan C: Explore the variation of MMP3, JNK, p38 MAPKs, and autophagy at the early stage of osteoarthritis. IUBMB Life 68: 293-302, 2016.

5. Andjelkov N, Elvenes J, Knutsen G and Johansen O: Beta-endorphin regulation of MAPKs in cultured human articular chondrocytes: MAPK inhibitors prevent the increase of IL-1 beta protein levels during beta-endorphin stimulation. Cell Commun Adhes 14: 1-8, 2007.

6. Ding L, Guo D and Homandberg GA: The cartilage chondrolytic mechanism of fibronectin fragments involves MAP kinases: Comparison of three fragments and native fibronectin. Osteoarthritis Cartilage 16: 1253-1262, 2008.

7. Roskoski RJ Jr: ERK1/2 MAP kinases: Structure, function, and regulation. Pharmacol Res 66: 105-143, 2012.

8. Fecher LA, Amaravadi RK and Flaherty KT: The MAPK pathway in melanoma. Curr Opin Oncol 20: 183-189, 2008.

9. Zhou Y, Liu SQ, Yu L, He B, Wu SH, Zhao Q, Xia SQ and Mei HJ: Berberine prevents nitric oxide-induced rat chondrocyte apoptosis and cartilage degeneration in a rat osteoarthritis model via AMPK and p38 MAPK signaling. Apoptosis 20: 1187-1199, 2015.

10. Kimura H, Yukitake H, Suzuki H, Tajima Y, Gomaibashi K, Morimoto S, Funabashi Y, Yamada K and Takizawa M: The chondroprotective agent ITZ-1 inhibits interleukin-1beta-induced matrix metalloproteinase- 13 production and suppresses nitric oxide-induced chondrocyte death. J Pharmacol Sci 110: 201-211, 2009.

11. Zhou J, Liao Y, Xie H, Liao Y, Liu H, Zeng Y and Li N: Pulsed electromagnetic field ameliorates cartilage degeneration by inhibiting mitogen-activated protein kinases in a rat model of osteoarthritis. Phys Ther Sport 24: 32-38, 2017.

12. Hayashi S, Nishiyama T, Miura Y, Fujishiro T, Kanzaki N Hashimoto S, Matsumoto T, Kurosaka M and Kuroda R: DcR3 induces cell proliferation through MAPK signaling in chondrocytes of osteoarthritis. Osteoarthritis Cartilage 19: 903-910, 2011 .

13. Shakibaei M, Mobasheri A and Buhrmann C: Curcumin synergizes with resveratrol to stimulate the MAPK signaling pathway in human articular chondrocytes in vitro. Genes Nutr 6: 171-179, 2011.

14. Lin M, Li X, Liu X, Lin Y, Zeng J, Liu J, Guo J, Liang Y, Tu X and Liu Q: Effect of warm sparse-dense waves on chondrocytic apoptosis regulation of gene expression. J Trad Chin Orthop Traumatol 24: 11-14, 2012 (In Chinese).

15. Panicker S, Borgia J, Fhied C, Mikecz K and Oegema TR: Oral glucosamine modulates the response of the liver and lymphocytes of the mesenteric lymph nodes in a papain-induced model of joint damage and repair. Osteoarthritis Cartilage 17: 1014-1021, 2009.

16. Li X, Lang W, Ye H, Yu F, Li H, Chen J, Cai L, Chen W, Lin R, Huang Y and Liu X: Tougu Xiaotong capsule inhibits the tidemark replication and cartilage degradation of papain-induced osteoarthritis by the regulation of chondrocyte autophagy. Int J Mol Med 31: 1349-1356, 2013.

17. Yan D, Liu TX, Liu BY, Wang L, Qian ZH, Cheng XG and Li KC: Effects of structural changes in subchondral bone on articular cartilage in a beagle dog model. Biomed Environ Sci 30: 194-203, 2017

18. Mankin HJ, Dorfman H, Lippiello L and Zarins A: Biochemical and metabolic abnormalities in articular cartilage from osteo-arthritic human hips. II. Correlation of morphology with biochemical and metabolic data. J Bone Joint Surg Am 53: 523-537, 1971.
19. Chen J, Liu G, Weng X, Liu F, Lin P, Li H, Chen W, Huang Y, Liu X, Ye H and Li X: Tougu Xiaotong formula induces chondrogenic differentiation in association with transforming growth factor- $\beta 1$ and promotes proliferation in bone marrow stromal cells. Int J Mol Med 35: 747-754, 2015.

20. Livak KJ and Schmittgen TD: Analysis of relative gene expression data using real-time quantitative PCR and the 2(-Delta Delta C (T)) method. Methods 25: 402-408, 2001.

21. Zhang C, Yu L, Zhou Y, Zhao Q and Liu SQ: Chitosan oligosaccharides inhibit IL-1 $\beta$-induced chondrocyte apoptosis via the P38 MAPK signaling pathway. Glycoconj J 33: 735-744, 2016.

22. Chan BY, Fuller ES, Russell AK, Smith SM, Smith MM, Jackson MT, Cake MA, Read RA, Bateman JF, Sambrook PN and Little CB: Increased chondrocyte sclerostin may protect against cartilage degradation in osteoarthritis. Osteoarthritis Cartilage 19: 874-885, 2011.

23. Huang JG, Xia C, Zheng XP, Yi TT, Wang XY, Song G and Zhang B: 17beta-Estradiol promotes cell proliferation in rat osteoarthritis model chondrocytes via PI3K/Akt pathway. Cell Mol Biol Lett 16: 564-575, 2011.

24. Lin M, Li XH, Lin YH, Lin YQ, Liu QH, Gao J, Zeng DH and Ke D: Effect of different warm dense-sparse waves on cartilage pathological changes of knee osteoarthritis. J Fujian Univ Trad Chin Med 26: 38-42, 2016 (In Chinese).

25. Bijlsma JW, Berenbaum F and Lafeber FP: Osteoarthritis: An update with relevance for clinical practice. Lancet 377: 2115-2126, 2011.

26. Gloth FR III: Pharmacological management of persistent pain in older persons: Focus on opioids and nonopioids. J Pain 12 (Suppl 3): S14-S20, 2011

27. Schnitzer TJ, Hochberg MC, Marrero CE, Duquesroix B Frayssinet $\mathrm{H}$ and Beekman M: Efficacy and safety of naproxcinod in patients with osteoarthritis of the knee: A 53-week prospective randomized multicenter study. Semin Arthritis Rheum 40: 285-297, 2011.

28. Dostrovsky NR, Towheed TE, Hudson RW and Anastassiades TP: The effect of glucosamine on glucose metabolism in humans: A systematic review of the literature. Osteoarthritis Cartilage 19: 375-380, 2011.

29. Health Quality Ontario: Arthroscopic lavage and debridement for osteoarthritis of the knee: An evidence-based analysis. Ont Health Technol Assess Ser 5: 1-37, 2005.

30. Fu MY and Zhang ZL: Knee osteoarthritis treated with acupuncture at the points selected according to syndrome differentiation: A randomized controlled trial. Zhongguo Zhen Jiu 31: 1062-1066, 2011 (In Chinese)

31. Ahsin S, Saleem S, Bhatti AM, Iles RK and Aslam M: Clinical and endocrinological changes after electro-acupuncture treatment in patients with osteoarthritis of the knee. Pain 147: 60-66, 2009.

32. Tan C, Wang J, Feng W, Ding W and Wang M: Preliminary correlation between warm needling treatment for knee osteoarthritis of deficiency-cold syndrome and metabolic functional genes and pathways. J Acupunct Meridian Stud 3: 173-180, 2010.

33. Li XH, Wu MX, Ye HZ, Chen WL, Lin JM, Zheng LP and Liu XX: Experimental study on the suppression of sodium nitroprussiate-induced chondrocyte apoptosis by Tougu Xiaotong Capsule (透骨消痛胶囊)-containing serum. Chin J Integr Med 17: 436-443, 2011 (In Chinese).

34. Rosenzweig DH, Ou SJ and Quinn TM: P38 mitogen-activated protein kinase promotes dedifferentiation of primary articular chondrocytes in monolayer culture. J Cell Mol Med 17: 508-517, 2013.

35. Wang X, Li F, Fan C, Wang C and Ruan H: Effects and relationship of ERK1 and ERK2 in interleukin-1 $\beta$-induced alterations in MMP3, MMP13, type II collagen and aggrecan expression in human chondrocytes. Int J Mol Med 27: 583-589, 2011.

36. Hashimoto S, Nishiyama T, Hayashi S, Fujishiro T, Takebe K, Kanzaki N, Kuroda R and Kurosaka M: Role of p53 in human chondrocyte apoptosis in response to shear strain. Arthritis Rheum 60: 2340-2349, 2009

37. Karnoub AE and Weinberg RA: Ras oncogenes: Split personalities. Nat Rev Mol Cell Biol 9: 517-531, 2008. 